\title{
A Distributionally Robust Power Dispatch Model for Active Distribution Network
}

\author{
Huihua Zhuang ${ }^{1}$, Huimin Zhuang ${ }^{2, *}$ \\ ${ }^{1}$ Locomotive and rolling stock Department, Kunming Railway Vocational and Technical College, Kunming, 650217, China \\ ${ }^{2}$ School of Control Engineering, Chengdu University of Information Technology, Chengdu, 610225, China
}

\begin{abstract}
The uncertainty of distribution network operation is increasing with the integration of large-scale renewable distributed generation (DG) units. To reduce the conservativeness of traditional robust optimization (RO) solutions, a data-driven robust optimal approach, which incorporates the superiority of both stochastic and robust approaches, is employed to solve the dispatch model in this paper. Firstly, a deterministic optimal dispatching model is established with the minimum total operation cost of distribution network; secondly, a two-stage distributed robust dispatching model is constructed based on the historical data of renewable-generators output available. The first stage of the model aims at finding optimal values under the basic prediction scenario. In the second stage, the uncertain probability distribution confidence sets with norm-1 and norm- $\infty$ constraints are integrated to find the optimal solution under the worst probability distribution. The model is solved by column-and-constraint generation (CCG) algorithm. Numerical simulation on the IEEE 33-bus system has been performed. Comparisons with the traditional stochastic and robust approaches demonstrate the effectiveness of the proposal.
\end{abstract}

\section{Introduction}

With the increasing number of the DGs, the traditional distribution network is going to be gradually transformed into the active distribution network (ADN), which is capable of coordinating DGs, energy storage systems (ESS), demand-side response to keep the distribution network operate in security and economy [1].

However, the active power output of distributed renewable generators (DRGs), such as photovoltaic (PV) and wind power generation (WT) units, may fluctuate with the changing weather conditions and is hard to predict accurately. To cope with the uncertainties brought by DRGs in power dispatch, a variety of approaches with different stochastic variables and constraints embedded have been employed extensively, such as stochastic optimization (SO) [2-3] and robust optimization (RO) [4-5]. In SO, random variables with a known probability distribution are generally used to account for the output of DRGs. Compared with SO, RO has the following upsides: more computationally tractable and easier to obtain empirical or predictive knowledge required by the uncertainty set of DRG output. However, RO tends to make more conservative dispatch decisions, and being robust to unnecessary situations could even lead to infeasibilities on some occasion.

Distributed Robust Optimization (DRO) incorporates the superiority of SO and RO to find the worst probability distribution in a range of known confidence sets. Traditional distributed robust optimization (TDRO) mainly applies Wasserstein scalar [6] or deterministic or uncertain first-order and second-order moments to construct probability distribution sets, so as to form complex NP problems for optimization solution [7]. But its solving process is very complex. In practice, a large number of DG historical data can be used to construct a series of possible probability distribution, which can be constrained by the sets of norm-1 and norm- $\infty$. This method is known as data-driven optimization [8-9], which neither require probabilistic distribution assumption nor need dualization, and thus it is less computational burden. Recently, this approach is adopted to solve the unit commitment problem [10-11], but has not been employed for power dispatch of distribution network to our best knowledge.

This paper proposes a two-stage robust optimization model for the economic dispatch of ADNs based on data-driven. The main contributions of this work include:

(1) The first stage optimization aims at minimizing comprehensive operation cost of $\mathrm{ADN}$ based on the forecasted output of DRGs, where the charging \& discharging state of energy storage devices (ESD) and power of demand response (DR) are selected as control variables.

(2) In the second stage optimal model, norm-1 and

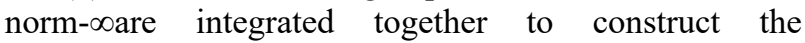
confidence set of uncertain probability distribution.

\footnotetext{
* Corresponding author: huimin@ cuit.edu.cn
} 


\section{Deterministic mathematical model}

\subsection{Objective function}

This optimization aims at the minimum total cost of ADN as follows:

$$
\begin{gathered}
\min \left(C_{1}+C_{2}+C_{3}\right) \\
C_{1}=C_{11}+C_{12}=\sum_{t=1}^{T} c_{t}^{0}\left(P_{t}^{0}+\sum_{i j \in \Omega_{\mathrm{b}}} r_{i j} I_{i j, t}^{2}\right) \Delta t \\
C_{2}=\sum_{t=1}^{T} \sum_{i=1}^{n}\left[\left(a_{i} P_{i, t}^{\mathrm{G}}+b_{i}\right)+\left(k_{i}^{\mathrm{c}} P_{i, t}^{\mathrm{c}}+k_{i}^{\mathrm{d}} P_{i, t}^{\mathrm{d}}\right)\right] \Delta t \\
C_{3}=\sum_{t=1}^{T} \sum_{i=1}^{n} k_{i}^{\mathrm{dr}}\left|P_{i, t}^{\mathrm{dr}}-P_{i, t}^{\mathrm{dr} *}\right| \Delta t
\end{gathered}
$$

Where $C_{11}$ represents the cost of purchasing electricity from the main network, $C_{12}$ is the total powerloss cost of the distribution network, $C_{2}$ denotes the generation cost of the dispatchable distributed energy resources, including gas turbines and ESDs, $C_{3}$ is the cost for scheduling DRs. $T, \Delta t$ are respectively the number of scheduling time intervals and the time interval; $c_{t}^{0}, P_{t}^{0}$ are respectively the electric price and the active power injected into the ADN from the main network at the period $t ; n$ is the number of nodes in ADN, $\boldsymbol{\Omega}_{\mathrm{b}}$ is the set of buanches, $r_{i j}$ is the resistance of branch $i j, I_{i j, t}$ is the current flowing through branch $i j$ at the period $t ; P_{i, t}{ }^{\mathrm{G}}$ is the output of the gas turbine located the node $i$ and $a_{i}, b_{i}$ are its coefficients of generation cost; $P_{i, t}{ }^{\mathrm{c}}$ and $P_{i, t}{ }^{\mathrm{d}}$ are respectively the charging power and discharging power of the ESD located the node $i$ at $t$, and $k_{i}{ }^{\mathrm{c}}$ and $k_{i}^{\mathrm{d}}$ are respectively its charging cost coefficient and discharging one; $P_{i, t}{ }^{\mathrm{dr}}$ and $P_{i, t}{ }^{\mathrm{dr} *}$ are respectively scheduled active power and original one of the DR located the node $i$ at the period $t$, and $k_{i}^{\mathrm{dr}}$ is its unit scheduling cost.

\subsection{System Constraints}

1) Power flow constraints

The equations representing the steady-state operation of a radial DN are shown in (2)[12]:

$$
\begin{gathered}
P_{j, t}=\sum_{k \in \delta(j)} P_{j k, t}-\sum_{i \in \pi(j)}\left(P_{i j, t}-\tilde{I}_{i j, t} r_{i j}\right), \vee j \in \boldsymbol{\Omega}_{\mathrm{n}} \\
Q_{j, t}=\sum_{k \in \delta(j)} Q_{j k, t}-\sum_{i \in \pi(j)}\left(Q_{i j, t}-\tilde{I}_{i j, t} x_{i j}\right), \vee j \in \boldsymbol{\Omega}_{\mathrm{n}} \\
\tilde{V}_{j, t}=\tilde{V}_{i, t}-2\left(P_{i j, t} r_{i j}+Q_{i j, t} x_{i j}\right)+\tilde{I}_{i j, t}\left(r_{i j, t}^{2}+x_{i j, t}^{2}\right), \vee i j \in \boldsymbol{\Omega}_{\mathrm{b}} \\
P_{i j, t}^{2}+Q_{i j, t}^{2}=\tilde{V}_{i, t} \tilde{I}_{i j, t}, \vee i j \in \boldsymbol{\Omega}_{\mathrm{b}}
\end{gathered}
$$

Where $\tilde{I}_{i j, t}=I_{i j, t}^{2}, \tilde{V}_{i, t}=V_{i, t}^{2}$. $\boldsymbol{\Omega}_{\mathrm{n}}$ is the set of nodes, $P_{i j, t}$, $Q_{i j, t}$ are respectively active power flow and reactive power flow of branch $i j$ at the period $t, \delta(j)$ is the set of branch terminal nodes with $j$ as the head node, $\boldsymbol{\pi}(j)$ is the set of branch head nodes with $j$ as the terminal node, $x_{i j}$ is the reactance of branch $i j, V_{i, t}, V_{j, t}$ are respectively the voltage amplitude of the node $i$ and $j$ at $t, P_{j, t}$ and $Q_{j, t}$ are respectively active power and reactive power injected into the node $j$ at the period $t$, which are expressed as (4):

$$
\begin{aligned}
& P_{j, t}=P_{j, t}^{\mathrm{G}}+P_{j, t}^{\mathrm{W}}+s_{j, t}^{\mathrm{d}} P_{j, t}^{\mathrm{d}}-s_{j, t}^{\mathrm{c}} P_{j, t}^{\mathrm{c}}-P_{j, t}^{\mathrm{L}} \\
& Q_{j, t}=\lambda_{j}^{\mathrm{G}} P_{j, t}^{\mathrm{G}}+Q_{j, t}^{\mathrm{cp}}-\lambda_{j}^{\mathrm{L}} P_{j, t}^{\mathrm{L}}
\end{aligned}
$$

Where $P_{j, t}{ }^{\mathrm{W}}, P_{j, t}^{\mathrm{L}}$ are respectively the output of WT and the active power of load located at the node $j$ at the period $t, Q_{j, t}{ }^{\mathrm{cp}}$ is the reactive power injected by the reactive power compensator located at the node $j$ at the period $t, \lambda_{j} \mathrm{G}$ and $\lambda_{j}^{\mathrm{L}}$ are respectively the tangent of power-factor angel of gas turbine and load located at the node $j$, WT operates in unit power factor in the paper. $s_{i, t}^{\mathrm{c}}, s_{i, t}^{\mathrm{d}}$ are bool variables, if $s_{i, t}^{\mathrm{c}}=1$, the ESD is extracting power from the ADN and if $s_{i, t}^{\mathrm{d}}=1$, the ESD is injecting power into the ADN.

The equation (3) is hard to be solved effectively due to its nonlinearity. By using the second-order cone relaxation technique[13], this equation is transformed into inequality constraint (5) which is of convexity.

$$
\left\|\begin{array}{c}
2 P_{i j, t} \\
2 Q_{i j, t} \\
\tilde{I}_{i j, t}-\tilde{V}_{i, t}
\end{array}\right\|_{2} \leq \tilde{I}_{i j, t}+\tilde{V}_{i, t}
$$

2) Operational constrains of the network

In order to ensure the safe operation of distribution network, the voltage of each node and the current of each branch should not exceed their limits as follows:

$$
V_{\min }^{2} \leq \tilde{V}_{i, t} \leq V_{\max }^{2}, \quad \tilde{I}_{i j, t} \leq I_{i j, \max }^{2}
$$

Where $V_{\max }, V_{\min }$ are respectively the upper and lower limits of voltage, $I_{i j \text {, max }}$ is the maximum current in the branch $i j$.

3) Operational constrains of gas turbines

The generation and ramping limitations are included:

$$
\begin{aligned}
& P_{i, \text { min }}^{\mathrm{G}} \leq P_{i, t}^{\mathrm{G}} \leq P_{i, \text { max }}^{\mathrm{G}} \\
& -r_{i}^{\mathrm{d}} \Delta t \leq P_{i, t}^{\mathrm{G}}-P_{i, t-1}^{\mathrm{G}} \leq r_{i}^{\mathrm{u}} \Delta t
\end{aligned}
$$

Where $P_{i, \text { max }}^{\mathrm{G}}, P_{i, \text { min }}^{\mathrm{G}}, r_{i}^{\mathrm{u}}, r_{i}^{\mathrm{d}}$ are respectively maximum and minimum active output and ramp-up rate and rampdown rate of the gas turbine located at the node $i$.

4) Operational constraints of ESD

For the ESD at node $i$, the operating constraints are as follows during the period $t$ :

$$
\begin{aligned}
& 0 \leq P_{i, t}^{\mathrm{c}} \leq s_{i, t}^{\mathrm{c}} P_{i, \max }^{\mathrm{c}}, \quad 0 \leq P_{i, t}^{\mathrm{d}} \leq s_{i, t}^{\mathrm{d}} P_{i, \text { max }}^{\mathrm{d}} \\
& E_{i, t+1}=E_{i, t}+\left(\eta_{\mathrm{c}} P_{i, t}^{\mathrm{c}}-P_{i, t}^{\mathrm{d}} / \eta_{\mathrm{d}}\right) \Delta t \\
& E_{i, \text { min }} \leq E_{i, t} \leq E_{i, \text { max }}, \quad s_{i, t}^{\mathrm{c}}+s_{i, t}^{\mathrm{d}} \leq 1
\end{aligned}
$$

Where $E_{i, t}$ denotes state of charge, $\eta_{\mathrm{c}}$ and $\eta_{\mathrm{d}}$ are respectively power extraction and injection efficiency, $P_{i, \text { max }}{ }^{\mathrm{c}}, P_{i, \text { max }} \mathrm{d}, E_{i, \text { max }} 、 E_{i, \text { min }}$ are respectively maximum power extraction capacity, maximum power injection capacity, upper and lower limits of energy storage.

5)DR constraints

Here, assuming DRs are the shifting loads which satisfy the constraints:

$$
\sum_{t=1}^{T} P_{i, t}^{\mathrm{dr}} \Delta t=D_{i}, \quad D_{i, t}^{\mathrm{min}} \leq P_{i, t}^{\mathrm{dr}} \Delta t \leq D_{i, t}^{\mathrm{max}}
$$


Where $D_{i}, D_{i, \max }, D_{i, \min }$ are respectively the total electricity of DR during the dispatching cycle and its maximum and minimum values .

\section{Data-driven two-stage robust optimal model}

In the last section, the optimization model is only conducted under a predicted DRG-output curve over multiple time periods. To address the uncertain generation output of RES, a two-stage robust optimal dispatch model is established to keep ADN operate safely under any cases.

Firstly, the optimization variables are classified into two parts: $\boldsymbol{x}=\left[\begin{array}{lll}s_{t}^{\mathrm{c}} & s_{t}^{\mathrm{d}} & \boldsymbol{P}_{t}^{\mathrm{dr}}\end{array}\right]$ and $\boldsymbol{y}=\left[\begin{array}{lll}\boldsymbol{P}_{t}^{\mathrm{G}} & \boldsymbol{P}_{t}^{\mathrm{c}} & \boldsymbol{P}_{t}^{\mathrm{d}}\end{array}\right] \cdot \boldsymbol{x}$ does not change frequently with fluctuation of DRGoutput. $\boldsymbol{y}$ can be flexible with the revealed uncertainty. Subsequently, the deterministic optimal scheduling model can be formulated as a general problem:

$$
\begin{gathered}
\min _{x, y} \boldsymbol{a}^{T} \boldsymbol{x}+\boldsymbol{b}^{\boldsymbol{T}} \boldsymbol{y} \\
\text { s.t. } \quad \boldsymbol{A} \boldsymbol{x} \leq \boldsymbol{d}_{1} \\
\boldsymbol{B y}=\boldsymbol{e}, \quad \boldsymbol{C} \boldsymbol{y} \leq\left[\begin{array}{ll}
\boldsymbol{d}_{2} & \zeta
\end{array}\right]^{T} \\
\boldsymbol{D x}+\boldsymbol{G} \boldsymbol{y}=\boldsymbol{g}, \quad\|\boldsymbol{J} \boldsymbol{x}+\boldsymbol{H} \boldsymbol{y}+\boldsymbol{h}\|_{2} \leq \boldsymbol{u}^{\mathrm{T}} \boldsymbol{x}+\boldsymbol{v}^{\mathrm{T}} \boldsymbol{y}+\boldsymbol{w}
\end{gathered}
$$

Where $\zeta$ is the vector of DRG-output, $\boldsymbol{a}, \boldsymbol{b}, \boldsymbol{A}, \boldsymbol{d}_{1}, \boldsymbol{B}$, $\boldsymbol{C}, \boldsymbol{e}, \boldsymbol{d}_{2}, \boldsymbol{G}, \boldsymbol{g}, \boldsymbol{D}, \boldsymbol{J}, \boldsymbol{H}, \boldsymbol{h}, \boldsymbol{u}, \boldsymbol{v}, \boldsymbol{w}$ are matrix/vector form with respect to the original model.

According to the model addressed above, a two-stage framework involving uncertainties is established. In the first stage, the vector $\boldsymbol{x}$ is solved to minimize the total cost of ADN under the initial scenario $\zeta_{0}$. In the second stage, the worst probability distribution of DRG output is obtained by optimizing the expected value of the cost respective of $\boldsymbol{y}$ when the probability distribution function of uncertainties $P(\zeta)$ is known. The general stochastic power dispatch model is formulated as follows:

$$
\min _{x \in X, y_{0} \in Y\left(x, \zeta_{0}\right)}\left\{\boldsymbol{a}^{\boldsymbol{T}} \boldsymbol{x}+\boldsymbol{b}^{\boldsymbol{T}} \boldsymbol{y}_{0}+\max _{P(\zeta) \in \Omega} \mathrm{E}_{\mathrm{P}}\left[\boldsymbol{b}^{\boldsymbol{T}} \boldsymbol{y}\right]\right\}
$$

Where $\mathbf{X}$ is the feasible region constructed by (11), $\mathbf{Y}\left(\boldsymbol{x}, \boldsymbol{\zeta}_{0}\right)$ denotes the feasible region constructed by (12) and (13) under the initial scenario $\zeta_{0}$, Ep represents the expected value.

Because $P(\zeta)$ is difficult to obtain, $K$ discrete scenarios (e.g. $\left.\xi_{1}, \xi_{2}, \ldots, \xi_{K}\right)$ are screened out from $M$ samples and the corresponding probability is $\left(p_{1}, \ldots\right.$, $\left.p_{K}\right)$. However, the probability distribution may not be exact due to the limited information from the historical data. Therefore, the probability distribution of uncertainties is allowed to be arbitrary within a predefined confidence set constructed from the historical data. Thus, the proposed data-driven stochastic power dispatch optimization model aims to find the optimal solution under the worst-case probability distribution, such that

$$
\min _{x \in X, y_{0} \in Y\left(x, \zeta_{0}\right)}\left\{\boldsymbol{a}^{T} \boldsymbol{x}+\boldsymbol{b}^{T} \boldsymbol{y}_{0}+\max _{\left\{p_{k}\right\} \in \Omega} \sum_{k=1}^{K} p_{k} \min _{y_{k} \in Y\left(x, \zeta_{k}\right)} \boldsymbol{b}^{T} \boldsymbol{y}_{k}\right\}
$$

Two popular confidence sets based on norm-1 and norm- $\infty$ were presented for $\Omega$ in [14], which can be described as follow:

$$
\Omega=\left\{\left\{p_{k}\right\} \mid \begin{array}{l}
p_{k} \geq 0, \quad \sum_{k=1}^{K} p_{k}=1 \\
\left|\sum_{k=1}^{K}\right| p_{k}-p_{k}^{0}\left|\leq \theta_{1}, \quad \max \right| p_{k}-p_{k}^{0} \mid \leq \theta_{\infty}
\end{array}\right.
$$

Where $p_{k}^{0}$ is the initial probability of the $k$ th discrete scenario, $\theta_{1}$ and $\theta_{\infty}$ denote allowable probabilistic deviation limits under norm- 1 and norm- $\infty$ constraints, respectively. In [14], for $K$ scenarios from $M$ samples, the relationship between the number of historical data and $\theta$ is described as follows:

$$
\begin{gathered}
\operatorname{Pr}\left\{\sum_{k=1}^{K}\left|p_{k}-p_{k}^{0}\right| \leq \theta_{1}\right\} \geq 1-2 K e^{-2 M \theta_{1} / K}=\alpha_{1} \\
\operatorname{Pr}\left\{\max \left|p_{k}-p_{k}^{0}\right| \leq \theta_{\infty}\right\} \geq 1-2 K e^{-2 M \theta_{\infty}}=\alpha_{\infty}
\end{gathered}
$$

\section{Solution procedure}

The proposed two-stage data-driven optimization model generally can be solved by the standard column-andconstraint generation method (C\&CG) [15], which is implemented in a master-sub-problem framework: subproblem (SP) aims to find the critical scenario of the uncertain set for a given first-stage decision variable that provides an upper bound; then new variables and constraints are added to the master problem (MP) to obtain a lower bound. MP and SP are solved iteratively and stops until the gap between the upper and lower bounds is smaller than a pre-set convergence tolerance.

MP aims to relax the original optimization model and provide a lower bound, which is described as follows:

$$
\begin{array}{r}
\text { (MP) } \min _{x \in X, y_{0} \in Y\left(x, \zeta_{0}\right), y_{k} \in Y\left(x, \zeta_{k}\right), L} \boldsymbol{a}^{\boldsymbol{T}} \boldsymbol{x}+\boldsymbol{b}^{\boldsymbol{T}} \boldsymbol{y}_{0}+L \\
\left.L \geq \sum_{k=1}^{K} p_{k}^{m}\left(\boldsymbol{b}^{T} \boldsymbol{y}_{k}^{m}+\boldsymbol{c}^{T} \boldsymbol{\zeta}_{k}\right)\right]
\end{array}
$$

For a given first-stage variables $\boldsymbol{x}^{*}$ in the $m$-th iteration, a second-stage bi-level "max-min" model is set up to find the worst-case scenario, yielding :

$$
\text { (SP) } L\left(\boldsymbol{x}^{*}\right)=\max _{\left\{p_{k}\right\} \in \Omega} \sum_{k=1}^{K} p_{k} \min _{y_{k} \in Y\left(x^{*}, \zeta_{k}\right)} \boldsymbol{b}^{T} \boldsymbol{y}_{k}
$$

Because the sub-feasible regions $(\mathrm{Y} 1, \ldots, \mathrm{Y} K)$ are separable, lower-level model of the bi-level model can be further decomposed into $K$ independent models, which are solved in parallel. Let $h_{k}=\min _{y_{k} \in Y\left(x^{*}, \zeta_{k}\right)} \boldsymbol{b}^{T} \boldsymbol{y}_{k}$, the second-stage "max-min" problem is reformulated as

$$
L\left(\boldsymbol{x}^{*}\right)=\max \sum_{k=1}^{K} p_{k} h_{k}
$$

MP and the SP are solved iteratively until the given convergence criteria is satisfied.

\section{Numerical analysis}

In this section, the approach put forward in this paper has been applied for a modified IEEE 33-bus test system to illustrate the performance of the proposed model. The computational tasks were performed on a $2.9 \mathrm{GHz}$ personal computer with 16 GB RAM, and the proposed 
method was programmed in MATLAB R2016a where the mixed integer programming was solved using CPLEX 12.8 .

\subsection{Test system data}

In this modified 33-bus distribution network plotted in Fig. 1, PV and WT located at buses \#16 and \#31 respectively are both of $0.5 \mathrm{MW}$. A gas turbine (GT) is installed at bus \#4. Two ESDs with $\eta_{\mathrm{c}}$ and $\eta_{\mathrm{d}}$ being both 0.9 are installed at bus $\# 17$ and \#29 respectively. The loads at bus \#25 and \#32 are both DR with unit scheduling cost being 0.32 yuan $/ \mathrm{kWh}$. All power factors are 0.95 . The safe range of voltage is from $0.95 \mathrm{pu}$ to $1.05 \mathrm{Pu}$, and the upper and lower limits of line current are $4 \mathrm{pu}$ and $-4 \mathrm{pu}$, respectively. The maximum power injected by the main network is $5 \mathrm{MW}$. Other parameters are seen in table 1,2. The forecasted load demand and DRG output over 24 hours are depicted in figures 2, where the DRG output is assumed to follow a multivariate normal distribution with the variance equivalent to $1 / 4$ of the mean value.

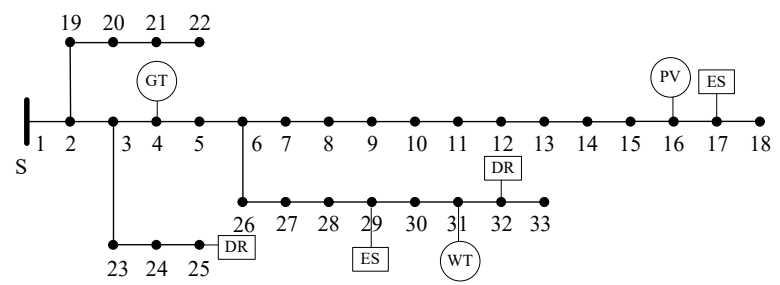

Figure 1. Modified IEEE33-bus distribution network

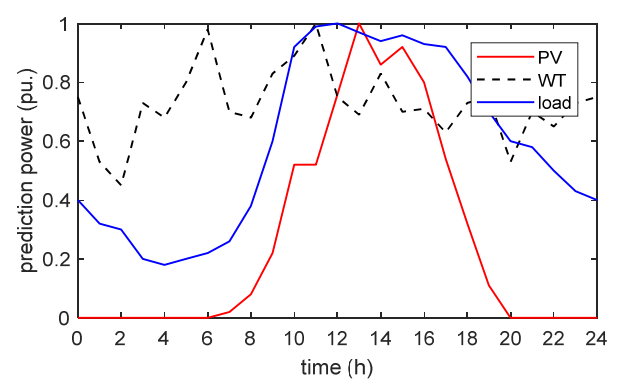

Figure 2. Prediction value of RESs and load

Table 1. Parameters of ESD and GT

\begin{tabular}{|c|c|c|c|c|c|c|}
\hline \multirow{3}{*}{ ESD } & $\begin{array}{c}P_{\max }^{\mathrm{c}} \\
(\mathrm{kW})\end{array}$ & $\begin{array}{c}P_{\max } \mathrm{d} \\
(\mathrm{kW})\end{array}$ & $\begin{array}{c}E_{\max } \\
(\mathrm{kWh})\end{array}$ & $\begin{array}{c}E_{\min } \\
(\mathrm{kWh})\end{array}$ & $\begin{array}{c}k^{\mathrm{c}}(Y / \\
\mathrm{kWh})\end{array}$ & $\begin{array}{c}K^{\mathrm{d}}(Y / \\
\mathrm{kWh})\end{array}$ \\
\cline { 2 - 7 } & 400 & 400 & 1200 & 120 & 0.4 & 0.6 \\
\hline \multirow{3}{*}{$\mathrm{GT}$} & $\begin{array}{c}P_{\max }{ }^{\mathrm{G}} \\
(\mathrm{kW})\end{array}$ & $\begin{array}{c}P_{\min }{ }^{\mathrm{G}} \\
(\mathrm{kW})\end{array}$ & $\begin{array}{c}r^{\mathrm{d}} \\
(\mathrm{kWh})\end{array}$ & $\begin{array}{c}r^{\mathrm{u}} \\
(\mathrm{kWh})\end{array}$ & $\begin{array}{c}a(¥ / \\
\mathrm{kWh})\end{array}$ & $\begin{array}{c}b(Y / \\
\mathrm{kWh})\end{array}$ \\
\cline { 2 - 7 } & 400 & 0 & 1200 & 0 & 0.69 & 0.69 \\
\hline
\end{tabular}

Table 2. TOU prices

\begin{tabular}{|l|c|}
\hline Time intervals & $\begin{array}{c}\text { Electricity price } \\
(\Psi / \mathrm{kWh})\end{array}$ \\
\hline 10:00-15:00, 18:00-21:00 & 1.32 \\
\hline 7:00-10:00,15:00-18:00, 21:00-23:00 & 0.83 \\
\hline 23:00-7:00 & 0.37 \\
\hline
\end{tabular}

\subsection{Optimization results and analysis}

We randomly generate 1000 samples by Monte Carlo simulation to simulate the set of the historical data. Let $\alpha_{1}=0.2, \alpha_{\infty}=0.8$. The proposed method in this paper is applied to acquire the optimal power dispatch scheme. The output of each controllable resource is depicted in figure 3 and the corresponding cost is shown in table 3. From figure 3, it sees that two ESDs are charged during the valley-load period from 00:00 to 06:00 at night, and discharge to $\mathrm{ADN}$ during the peak-load period from 10:00 to 18:00 in the daytime, which indicates ESDs play an important role in peak shaving and valley filling.

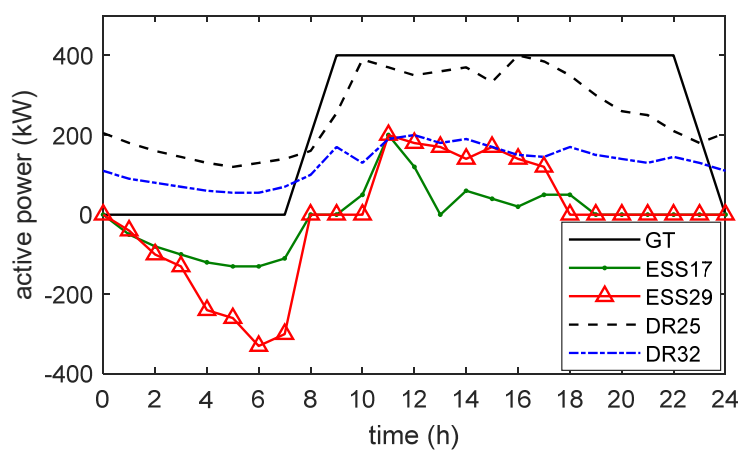

Figure 3. Robust optimal scheduling

Table 3. Power dispatch cost

\begin{tabular}{|c|c|c|c|c|c|}
\hline \multicolumn{5}{|c|}{ Individual cost (Yuan) } & \multirow{2}{*}{$\begin{array}{l}\text { Total cost } \\
\text { (Yuan) }\end{array}$} \\
\hline$C_{11}$ & $C_{12}$ & $C_{21}$ & $C_{22}$ & $C_{3}$ & \\
\hline 34971.8 & 1007.3 & 4800.0 & 1608.5 & 326.1 & 42713.7 \\
\hline
\end{tabular}

The voltage profile of node 33 during 24 hours is depicted in figure 4 , which shows that the presented optimal dispatch scheme ensures that ADN can still operate safely in worst scenario of uncertainties.

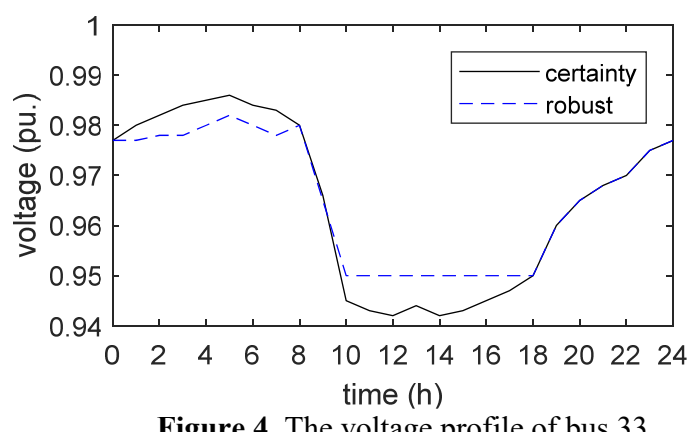

Figure 4. The voltage profile of bus 33

\subsubsection{Comparison under different confidence set}

$M$ is 1000 , and the total cost is listed in table 4 under different confidence set. Table 4 shows that the total cost goes up with the increase of $\alpha_{1}$ or $\alpha_{\infty}$. With increase of confidence set, the uncertainty will rise, and more controllable resources need to be dispatched, which leads to larger total cost.

Table 4. Total cost (Yuan) in case of different confidence set

\begin{tabular}{|c|c|c|c|}
\hline \multirow{2}{*}{$\boldsymbol{\alpha}_{\mathbf{1}}$} & \multicolumn{3}{|c|}{$\boldsymbol{\alpha}_{\infty}$} \\
\cline { 2 - 4 } & 0.5 & 0.8 & 0.99 \\
\hline 0.2 & 42629.8 & 42713.7 & 42843.5 \\
\hline
\end{tabular}




\begin{tabular}{|c|c|c|c|}
\hline 0.5 & 42636.2 & 42765.4 & 42930.1 \\
\hline 0.99 & 42636.2 & 42773.1 & 43214.9 \\
\hline
\end{tabular}

\subsubsection{Comparison with Traditional Stochastic and Robust Approaches}

In this section, the propsed data-driven robust optimization (DRO) is compared with the traditional stochastic optimization ( $\mathrm{SO}$ ) and robust optimization (RO) methods. SO is preceded based on 10 scenarios with probability of each scenario being 0.1 . RO is tested with the fluctuation range being 0.25 times of predicted value. $\alpha_{1}=0.2, \alpha_{\infty}=0.8 .1000$ probability distribution scenarios are randomly generated to obtain the average and maximum values of network loss expectations as shown in Table 5.

Table 5. Comparison between different algorithms

\begin{tabular}{|c|c|c|c|}
\hline \multirow{2}{*}{ Method } & \multirow{2}{*}{$\begin{array}{c}\text { Optimal total } \\
\text { cost (Yuan) }\end{array}$} & \multicolumn{2}{|c|}{$\begin{array}{c}\text { Cost Expectation with } \\
\text { probability distribution } \\
\text { (Yuan) }\end{array}$} \\
\cline { 3 - 4 } & & average & maximum \\
\hline DRO & 42713.7 & 41615.7 & 43530.8 \\
\hline SO & 41950.0 & 42470.4 & 46579.5 \\
\hline RO & 47266.0 & 43267.7 & 49048.5 \\
\hline
\end{tabular}

As can be seen from Table 5, RO yields the highest network loss because it procede under the worst-case for all the possible realizations, while SO only copes with 10 scenarios, which leads to the lowest optimization results. Compared with RO and SO, DRO model achieves a better balance in economy and conservativeness. In addition, by comparing the expected values of probability-distribution cost, it can be seen that DRO obtains the lowest probability distribution expectation because of taking into account the uncertainty of scenarios, which shows DRO is of optimal economic performance and uncertainty adaptability.

\section{Conclusion}

Considering the uncertainty of DRG output, a datadriven robust optimal dispatch model is established in this paper for ADN with DGs, energy storage devices and demand response of loads. According to the historical data, a confidence set constrained by norm-1 and norm- $\infty$ is constructed for the probability distribution of the uncertainties. The proposed method aims to find an optimal solution under the worst probability distribution. Furthermore, linearization is used to simplify the constraints with the absolute term. Then C\&CG algorithm is employed to decompose the second-stage bi-level inner problem into several smallscale sub-problems which can be proceeded in parallel. The comparison with the traditional stochastic and robust approaches on IEEE 33-bus test system indicates that the proposed model can achieve better optimal solution and computational performance than traditional methods.

\section{References}

1. A. B. M. Shawkat Ali, Ed., Smart Grids: Opportunities, Developments, and Trends, Green Energy and Technology. New York, NY, USA: Springer (2013)

2. Z. S. Zhang, Y. Z. Sun, D. W. Gao et al., A Versatile Probability Distribution Model for Wind Power Forecast Errors and Its Application in Economic Dispatch, IEEE Transactions on Power Systems, vol. 28, no. 3, pp. 3114-3125, (2013)

3. W. Zheng, W. Wu, B. Zhang et al., Fully distributed multi-area economic dispatch method for active distribution networks, IET Generation, Transmission \& Distribution, vol.9, no. 12, pp. 1341-1351, (2015)

4. M. Bazrafshan, and N. Gatsis, Decentralized Stochastic Optimal Power Flow in Radial Networks With Distributed Generation, IEEE Transactions on Smart Grid, vol. 8, no. 2, pp. 787-801, (2017)

5. Liu Yixin, Guo Li, Wang Chengshan. Economic dispatch of microgrid based on two stage robust optimization, Proceedings of the CSEE, vol. 38, no. 14, pp. 4013-4022, (2018)

6. D. Bertsimas, X. V. Doan, K. Natarajan et al., Models for minimax stochastic linear optimization problems with risk aversion, Mathematics of Operations Research, vol. 35, no. 3, pp. 580-602, (2010)

7. G. Bayraksan, and D. K. Love, Data-driven stochastic programming using phi-divergences, the Operations Research Revolution, pp. 1-19: INFORMS, (2015)

8. Y. Zhang, S. Shen, and J. L. Mathieu, Distributionally robust chance constrained optimal power flow with uncertain renewables and uncertain reserves provided by loads, IEEE Trans. Power Syst., vol. 32, no. 2, pp. 1378-1388, (2017)

9. W. Wei, F. Liu, and S. Mei, Distributionally robust co-optimization of energy and reserve dispatch, IEEE Trans. Sustain. Energy, vol. 7, no. 1, pp. 289300, (2016)

10. P. Xiong, P. Jirutitijaroen, and C. Singh, A distributionally robust optimization model for unit commitment considering uncertain wind power generation, IEEE Trans. Power Syst., vol. 32, no. 7, pp. 39-49, (2017)

11. M. Khanabadi, Y. Fu, and L. Gong, A fully parallel stochastic multiarea power system operation considering large-scale wind power integration, IEEE Trans. Sustain. Energy, vol. 9, no. 1, pp. 138147, (2018)

12. J. F. Franco, M. J. Rider, M. Lavorato, and R. Romero, Optimal conductor size selection and reconductoring in radial distribution systems using a mixed-integer LP approach, IEEE Trans. Power Syst., vol. 28, no. 1, pp. 10-20, (2013)

13. M. Farivar and S. H. Low, Branch flow model: Relaxations and convexification-Part I, IEEE 
Trans. Power Syst., vol. 28, no. 3, pp. 2554-2564, (2013)

14. T Ding, Y Qingrun, L Xiyuan, etc. Duality-Free Decomposition Based Data-Driven Stochastic Security-Constrained Unit Commitment, IEEE Trans. On Sustainable Energy, vol. 10, no. 1, pp. 2825-2836, (2019)

15. B. Zeng and L. Zhao, Solving two-stage robust optimization problems using a column-andconstraint generation method, Oper. Res. Lett., vol. 41, no. 5, pp. 457-461, (2013) 